\title{
Zeeman effect in superconducting two-leg ladders: irrational magnetization plateaus and exceeding the Pauli limit.
}

\author{
G. Roux, ${ }^{1, *}$ S. R. White, ${ }^{2}$ S. Capponi, ${ }^{1}$ and D. Poilblanc ${ }^{1}$ \\ ${ }^{1}$ Laboratoire de Physique Théorique, UMR5152 Université Paul Sabatier, 31062 Toulouse, France. \\ ${ }^{2}$ Department of Physics and Astronomy, University of California, Irvine CA 92697, USA.
}

(Dated: October 18, 2018)

\begin{abstract}
The effect of a parallel magnetic field on superconducting two-leg ladders is investigated numerically. The magnetization curve displays an irrational plateau at a magnetization equal to the hole density. Remarkably, its stability is fundamentally connected to the existence of a well-known magnetic resonant mode. Once the zero-field spin gap is suppressed by the field, pairs acquire a finite momentum characteristic of a Fulde-Ferrell-Larkin-Ovchinnikov phase. In addition, $S^{z}=0$ triplet superconducting correlations coexist with singlet ones above the irrational plateau. This provides a simple mechanism in which the Pauli limit is exceeded as suggested by recent experiments.
\end{abstract}

PACS numbers: 71.10.Pm, 75.60.-d, 74.81.-g, 75.40.Mg

Ladder systems, which consists of two or more strongly coupled chains, have been widely studied because of their exotic properties 1]. In particular, two-leg ladders are Mott insulators at half filling, with a spin gap, and become superconducting under doping (and pressure) 2]. This doped spin-liquid is believed to be a paradigm for the Resonating Valence Bond mechanism of superconductivity proposed by Anderson [3]. Such a low-dimensional superconductor provides an appealing situation to study the role of a parallel magnetic field (Zeeman effect). In a singlet superconductor, the critical magnetic field which destroys pairing by splitting up the up and down spin Fermi surfaces is called Pauli limit. The possibility of exceeding this theoretical limit and/or stabilizing inhomogeneous superconductivity predicted by Fulde, Ferrell, Larkin and Ovchinnikov (FFLO) 4], have attracted strong interest in lowdimensional superconductors [5]. In particular, recent experiments suggest that Pauli limit is exceeded in the ladder material $\mathrm{Sr}_{14-x} \mathrm{Ca}_{x} \mathrm{Cu}_{24} \mathrm{O}_{41}$ (SCCO) [6, 7]. Furthermore, under magnetic field, insulating ladders can have plateaus for rational values of the magnetization, depending on the number of legs and on the interactions [8]. Doped low-dimensional strongly correlated systems such as doped spin chains [9, 10] have also proved to have interesting magnetization curves with plateaus at irrational values controlled by hole doping. Recently, Cabra et al. 11 have predicted such magnetization plateaus in doped Hubbard ladders by means of Abelian bosonization and a strong-coupling expansion. However, numerical evidence supporting such a scenario is lacking.

In this letter, we investigate doped two-leg ladders with a Zeeman coupling using the density matrix renormalization group (DMRG) 12, 13. . We show that, for isotropic couplings giving rise to a superconducting Luther-Emery phase [14, 15], a non-trivial plateau occurs for the finite magnetization value predicted in Yamanaka-OshikawaAffleck (YOA) theorem [16]. We give a simple physical argument to explain this behavior which is similar to the formation of the resonant magnetic mode encountered in the study of spin dynamics [17]. We finally discuss the evolution of the ground state properties under magnetic field. In particular, we focus on the persistence of superconductivity for magnetic fields larger than the Pauli limit. This persistence is associated with the emergence of Cooper pairs with a finite total momentum, a typical feature of FFLO phases. We also observed the coexistence of singlet and triplet pairing.

We describe the doped two-leg ladder using the $t-J$ model with the same couplings along the legs and between the legs. This model is believed to account for the unconventional superconductivity in lightly doped ladders, like the one observed in SCCO. We consider a static and uniform magnetic field $\mathbf{H}$ which couples to the spin degree of freedom via a Zeeman term. This corresponds to a magnetic field oriented parallel to the ladder plane to avoid orbital effect. Hence, we can write the Hamiltonian

$$
\begin{aligned}
\mathcal{H}= & -t \sum_{\langle i, j\rangle, s} \mathcal{P}_{G}\left[c_{i, s}^{\dagger} c_{j, s}+c_{j, s}^{\dagger} c_{i, s}\right] \mathcal{P}_{G} \\
& +J \sum_{\langle i, j\rangle}\left[\mathbf{S}_{i} \cdot \mathbf{S}_{j}-\frac{1}{4} n_{i} n_{j}\right]-g \mu_{B} \sum_{i} \mathbf{H} \cdot \mathbf{S}_{i}
\end{aligned}
$$

in which $\mathcal{P}_{G}$ is the Gutzwiller projector, $c_{i, s}^{\dagger}, \mathbf{S}_{i}$ and $n_{i}$ are respectively electron creation, spin and density operators at site $i$ and $s$ is the spin index. In what follows, the coupling constant $g \mu_{B}$ is absorbed in the definition of $\mathbf{H}$. We computed energies at fixed magnetization using the single center site method recently proposed by one of us which enabled us to have a discarded weight of order $10^{-7}-10^{-8}$ with 1600 kept states and a noise level of $10^{-6}$ 18. Energies are denoted by $E\left(n_{h}, S^{z}\right)$ with $n_{h}$ the number of holes and $S^{z}$ the total spin along the field. Open boundary conditions are used, $L$ stands for the ladder length and $\delta=n_{h} / N_{\text {sites }}$ is the hole doping $\left(N_{\text {sites }}=2 L\right)$. All data were computed with $J / t=0.5$ for which the model is known to have dominant superconducting correlations at low doping 14,15 . 


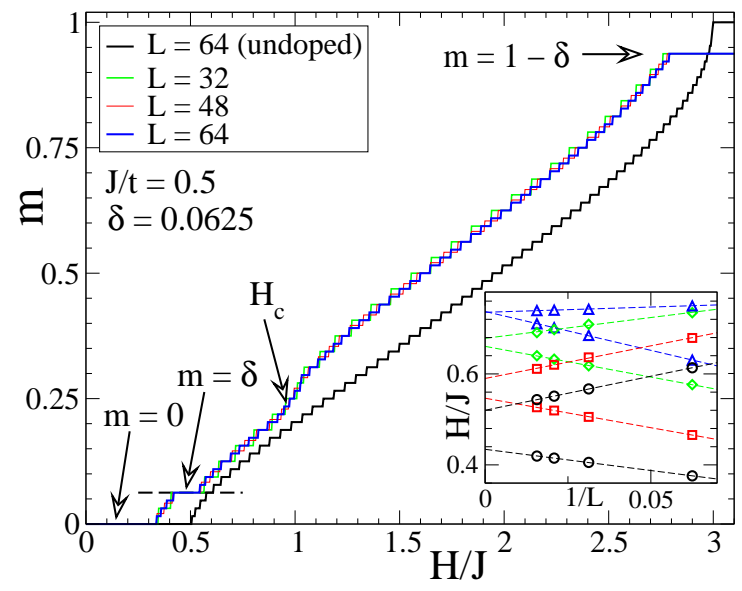

FIG. 1: (Color online) Magnetization curves at fixed hole density $\delta=0.0625$ for different system sizes. Three plateaus are visible for $m=0, \delta$ and $1-\delta . H_{c}$ is the superconducting critical field (see text) and results in a discontinuity of the slope in the magnetization curve. The undoped case is shown for comparison. Insert : Finite-size scaling of the two critical fields corresponding to the boundaries of the $m=\delta$ plateau for various densities $\delta=0.0625(\bigcirc), 0.125(\square), 0.1875(\diamond), 0.25(\triangle)$.

Yamanaka-Oshikawa-Affleck theorem - We first recall the topological constraints that govern the opening of gaps in the excitation spectrum of one-dimensional like systems [16]. In the case of doped two-leg ladders, YOA relation takes the simple form

$$
1-\delta \pm m \in \mathbb{Z}
$$

in which $m=2 S^{z} / N_{\text {sites }}$ is the total magnetization normalized so that it equals $1-\delta$ at saturation. It is a commensurability condition for either spin $\uparrow$ or $\downarrow$ but it introduces irrationality through $\delta$. For $\delta$ small enough, the relation can be rewritten

$$
m=\delta \quad \text { or } \quad m=1-\delta .
$$

The theorem states that: $(i)$ if the magnetization-doping relation is not satisfied, the low-energy spectrum can be either a continuum or gapped, but this latest case is possible only if $1-\delta \pm m=p / q \in \mathbb{Q}$ and is associated with a spontaneous breaking of the translational symmetry so that the ground state is $q$-fold degenerate, $(i i)$ if the relation is satisfied, a gap can open in the excitation spectrum. Note that for doped systems with charge and spin degrees of freedom, one sector can be gapped while the other remains gapless. This actually happens for superconducting two-leg ladders: the spin gap (related to the $m=0$ plateau) survives for arbitrary $\delta \neq 0$ but the theorem is still valid because a gapless charge mode appears simultaneously. This is the so-called Luther-Emery phase describing the system when $\mathbf{H}=0$.

Plateau phase diagram - The magnetization curve for a fixed hole density is given in FIG. 1 Three plateaus

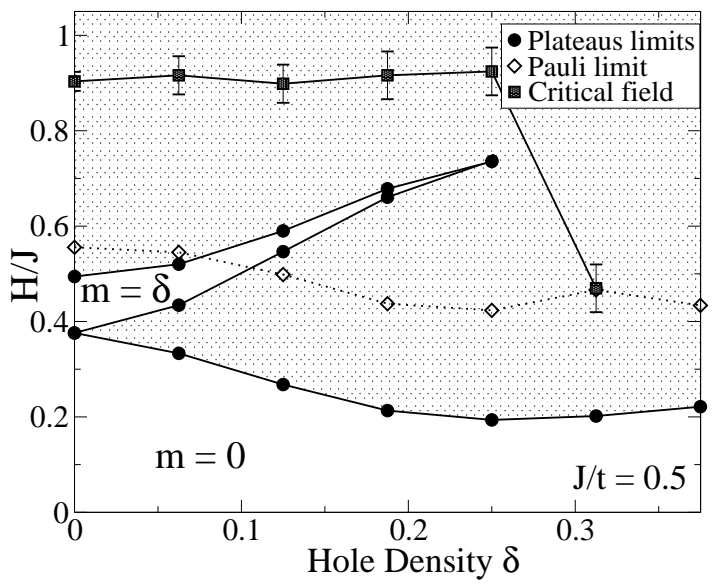

FIG. 2: Plateau phase diagram in the $(\mathbf{H}, \delta)$ plane. The expected Pauli limit $H_{p}$ (see text) is shown in dashed lines. The superconducting critical field $H_{c}$ is also shown (the error bars indicate the uncertainty in determining the transition).

are visible at $m=0, \delta$ and $1-\delta$. The first one corresponds to the well known spin gap. The second one is expected from the YOA relation and is the most interesting case. The third one simply corresponds to the full saturation of the spins. In addition to these plateaus, the magnetization curve displays a discontinuity in its slope at a magnetic field $H_{c}$ above the $m=\delta$ plateau. We will see that this critical field (consistent with a second order transition) is the superconducting critical field of the system. The critical fields of the transitions delimiting the plateaus have been extrapolated to the thermodynamic limit for various densities (see FIG. 1) and are gathered in FIG. 2 The $\delta \rightarrow 0$ limit has been taken with 2 holes and $L \rightarrow \infty$. The continuous behavior vs $\delta$ is consistent with a doping-dependent magnetization plateaus 10, 11]. The width of the $m=\delta$ plateau is much smaller than the width of the $m=0$ plateau and vanishes for $\delta \simeq 0.25$. In the $\delta \rightarrow 0$ limit, it is exactly the binding energy of the hole pair-magnon bound state previously discussed [17]. This bound state originates from the coupling of the magnon to the charge mode which leads to an abrupt decrease of the spin gap in the $\delta \rightarrow 0$ limit. Therefore, the formation of the $m=\delta$ plateau is based on a similar mechanism. To illustrate this, the local hole and spin densities in the ground state have been computed by DMRG and are displayed in FIG. 3 for increasing magnetization on a system with 6 holes. For the chosen parameters, holes are paired up and one can see three maxima in the hole density, which qualitatively correspond to each pair of holes (FIG. 3 $m=0$ ). When a single magnon is present in the system (FIG. 3 $m=0.021$ ), it preferably binds to a pair of holes. This is due to the fact that holes gain kinetic energy in a ferromagnetic environment. As more magnons are added, more bound states progressively form until $m=\delta$, i.e. when the number of magnons equals the number of hole 


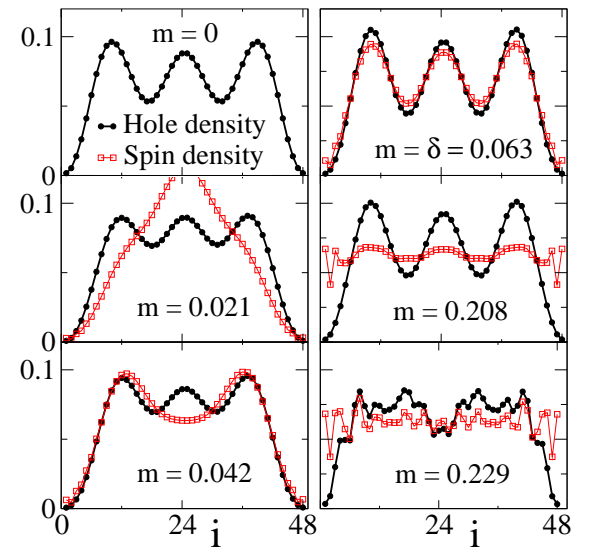

FIG. 3: (Color online) Evolution of local densities for increasing magnetization $m$ in a system with 6 holes and $L=48$. The spin density is normalized by $2 \delta / m$ so that spin and hole densities always have the same mean value.

pairs. Adding another magnon in this system costs a finite energy gap leading to the $m=\delta$ plateau. Note that other mechanisms could lead to plateaus in this system but they would correspond to different parameters in the Hamiltonian. By analyzing local densities beyond the $m=\delta$ plateau, one observes that hole pairs survive up to a magnetization $m \simeq 0.22$. Above the corresponding critical field, consistent with the value of $H_{c}$ in FIG. 1] the system behaves qualitatively like a gas of decoupled spinons and holons (FIG. B $m=0.229$ ).

To confirm the fact that $H_{c}$ is the superconducting critical field, we have first computed the pairing energy $\Delta_{p}$ as a function of magnetization using

$$
\begin{aligned}
\Delta_{p}\left(S^{z}\right)= & E\left(n_{h}-1, S^{z}+1 / 2\right)+E\left(n_{h}-1, S^{z}-1 / 2\right) \\
& -E\left(n_{h}, S^{z}\right)-E\left(n_{h}-2, S^{z}\right) .
\end{aligned}
$$

The results plotted at fixed density on FIG. 4 show that pairing survives far beyond the plateau. We will see that the superconducting correlations are also significant up to $H_{c}$. The $H_{c}$ line on FIG. 2 was evaluated from points where $\Delta_{p}\left(S^{z}\right)=0$ on a system with $L=64$. To estimate the Pauli limit in this system, we identify the condensation energy $\Delta_{p}^{0}$ at $\mathbf{H}=0$ to $\Delta_{p}\left(S^{z}=0\right)$. Comparing it with the Zeeman stabilization of two up-electrons gives the Pauli limit $H_{P}=\Delta_{p}^{0}$. Extrapolated results are shown in FIG. 22 The fact that the actual superconducting critical field $H_{c}$ is larger than $H_{P}$ suggests that the Pauli limit is exceeded in this system. We will see that this can be explained by the appearance of FFLO-like pairing above the $m=0$ plateau.

Evolution of the ground state - In order to have a better characterization of the nature of the ground state under a magnetic field, we have calculated superconducting correlations $\left\langle\Delta_{\sigma \sigma^{\prime}}^{\lambda}(r) \Delta_{\sigma \sigma^{\prime}}^{\lambda \dagger}(0)\right\rangle$ where $\lambda$ characterizes the pairing channel, having a discarded weight of order $10^{-6}$ with 1000 kept states. Both singlet and triplet channels

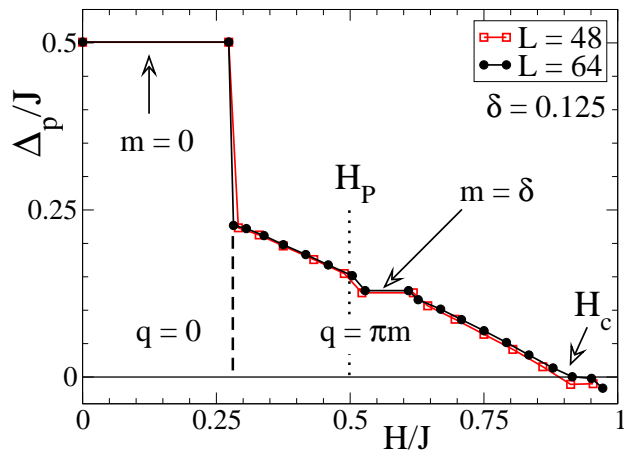

FIG. 4: (Color online) Pairing energy $\Delta_{p}$ (see definition in text) as a function of $\mathbf{H}$ for $\delta=0.125$. The dashed line indicates the transition to the FFLO state $(q=0 \rightarrow q=\pi m)$.
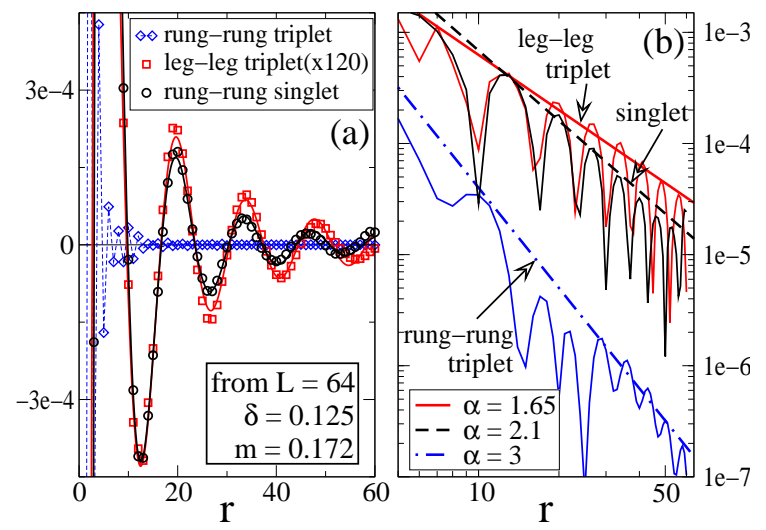

FIG. 5: (Color online) (a) Superconducting correlations in real space showing $q=\pi m$ oscillations for rung-rung singlet and leg-leg triplet $\left(S^{z}=0\right)$ correlations while rung-rung triplet $\left(S^{z}=+1\right)$ correlations decay faster (continuous lines are fits). (b) same in log-log scale showing the algebraic decaying with exponent $\alpha$ (straight lines are envelopes of fits).

have been studied for nearest-neighbor (NN) pairs that can be either along a rung (called "rung" pair) or on the same leg (called "leg" pair). When $\mathbf{H} \neq 0$, the four types of spin-pairing are inequivalent

$$
\begin{array}{ll}
\text { singlet: } & \Delta_{\uparrow \downarrow}^{s}(r)=c_{r \uparrow} c_{r+p, \downarrow}-c_{r \downarrow} c_{r+p, \uparrow} \\
\text { triplet: } & \left\{\begin{array}{c}
\Delta_{\uparrow \downarrow}^{t}(r)=c_{r \uparrow} c_{r+p \downarrow}+c_{r \downarrow} c_{r+p \uparrow} \\
\Delta_{\uparrow \uparrow}^{t}(r)=c_{r \uparrow} c_{r+p \uparrow} \text { and } \Delta_{\downarrow \downarrow}^{t}(r)=c_{r \downarrow} c_{r+p \downarrow}
\end{array}\right.
\end{array}
$$

in which $p$ determines the position of the partner (on same rung or same leg). In the $m=0$ plateau, two-leg superconducting ladders have anisotropic $d$-wave singlet pairing [14, 19]. Rung-leg correlations and leg-leg/rungrung correlations thus have an opposite sign in the singlet channel. Above the $m=0$ plateau, we found that both singlet and leg-leg $S^{z}=0$ triplet channels give rise to dominant correlations which long-range behavior is $\cos (q r) / r^{\alpha}$ (see FIG. 5). The wave vector of the oscillations varies as $q=\pi m$. This can be qualitatively understood by considering the Fermi momenta of paired electrons $k_{F}^{\uparrow, \downarrow}=\frac{\pi}{2}(1-\delta \pm m)$ on a single chain [20]. Cooper 

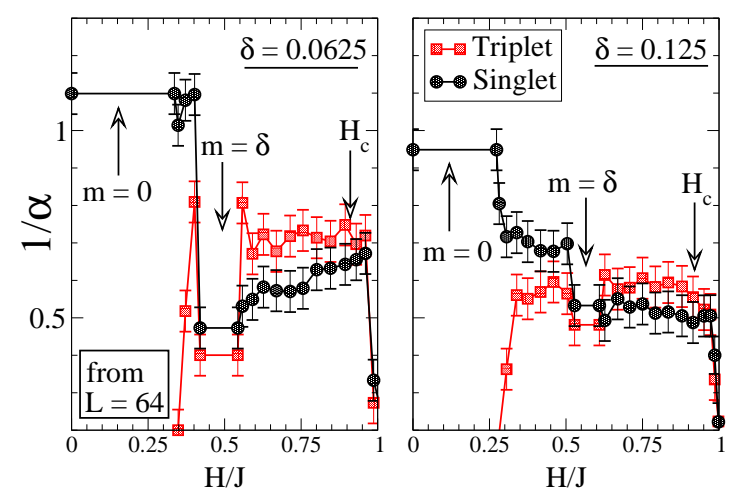

FIG. 6: (Color online) Inverse of the superconducting correlations exponent $\alpha$ as a function of $\mathbf{H}$ for two densities. Singlet and $S^{z}=0$ triplet correlations coexist above the $m=\delta$ plateau. Error bars are estimates of uncertainties from fitting.

pairs with $S^{z}=0$ are formed with a total momentum $q=k_{F}^{\uparrow}-k_{F}^{\downarrow}=\pi m$ characteristic of FFLO phases [4]. We extracted the exponent $\alpha$ to determine the strength of the correlations (see FIG. [6). In spite of the difficulty of extracting precisely $\alpha$, we found clear evidence that both channels are coexisting above the plateau (although, because of remaining finite size effects, we cannot conclude on the definite dominance of one w.r.t. the other [20]). Such a rich behavior emerging under a parallel magnetic field might be surprising at first sight. However, a strong field dependence of the superconducting correlations is in fact expected since pairing originates from magnetic fluctuations. In the irrational plateau, weaker superconducting correlations are found together with an anomaly in the compressibility (not shown). The latter could be explained from the finite energy gap needed to remove a pair of holes at fixed magnetization (leading to a deviation from the $m=\delta$ stability condition). Dominant charge density wave (CDW) correlations could then be expected in this plateau [20]. Note also that the overall magnitude of the triplet signal is about a hundred times smaller than the singlet one probably due to a more complex internal pairing structure which NN pairs only approximate. The leg-leg triplet pairing is symmetric vs the exchange of chains but further investigations have to be done to determine the precise orbital structure.

Experiments - Experiments in the superconducting phase of the ladder material SCCO are difficult because of high pressure and the presence of a chain subsystem. Transport experiments have found that the superconducting critical field is strongly anisotropic and that the Pauli limit is likely to be exceeded [6, 7]. We would like to point out that this behavior does not necessarily imply polarized triplet pairing at $\mathbf{H}=0$ or spin-orbit coupling. Indeed, this study suggests that ladder systems should have a wide FFLO phase explaining the exceeding of the Pauli limit. Also, possible observation of irrational magnetization plateaus could determine the hole doping $\delta$ in ladders. Note that a NMR study proposes $\delta \simeq 0.1$ in the superconducting phase 21].

Conclusion - In conclusion, we show that irrational magnetization plateaus predicted in 11] do appear in the study of the isotropic $t-J$ model on two-leg ladders. Furthermore, the system has a superconducting critical field larger than the Pauli limit which can be explained by the emergence of pairing with a total finite momentum $q=\pi m$ typical of a FFLO phase. This possible explanation for experiments does not resort to spin-orbit coupling nor triplet superconductivity at $\mathbf{H}=0$. In addition, under high magnetic field, we found the emergence of $S^{z}=0$ triplet pairing coexisting with singlet pairing.

Acknowledgments - We would like to thank Manfred Sigrist and Andreas Läuchli for very fruitful discussions. G. R. thanks IDRIS (Orsay, France) for use of supercomputer facilities. S. R. W. acknowledges the support of the NSF under grant DMR03-11843. We thank ANR for support.

* Electronic address: roux@irsamc.ups-tlse.fr

[1] E. Dagotto and T. M. Rice, Science 271, 618 (1996).

[2] M. Uehara et al., J. Phys. Soc. Jpn. 65, 2764 (1996). See also H. Mayaffre et al., Science 279, 345 (1998).

[3] P. W. Anderson, Science 235, 1196 (1987).

[4] P. Fulde and R. A. Ferrell, Phys. Rev. 135, A550 (1964); A. I. Larkin and Yu. N. Ovchinnikov, Sov. Phys. JETP 20, 762 (1965).

[5] T. Ishiguro, in High Magnetic Fields, edited by C. Berthier, L. P. Lévy, and G. Martinez, Lecture notes in physics Vol. 595 (Springer, 2002).

[6] D. Braithwaite et al., Solid State Com. 114, 533 (2000).

[7] T. Nakanishi et al., Phys. Rev. B 72, 054520 (2005).

[8] D. C. Cabra, A. Honecker, and P. Pujol, Phys. Rev. Lett. 79, 5126 (1997); Phys. Rev. B 58, 6241 (1998).

[9] H. Frahm, C. Sobiella, Phys. Rev. Lett. 83, 5579 (1999).

[10] D. C. Cabra et al., Phys. Lett. A 268, 418 (2000); Phys. Rev. B 63, 094406 (2001).

[11] D. C. Cabra, A. De Martino, P. Pujol, and P. Simon, Euro. Phys. Lett. 57, 402 (2002).

[12] S. R. White, Phys. Rev. Lett. 69, 2863 (1992); Phys. Rev. B 48, 10345 (1993).

[13] U. Schollwöck, Rev. Mod. Phys. 77, 259 (2005).

[14] C. A. Hayward et al., Phys. Rev. Lett. 75, 926 (1995).

[15] S. R. White, I. Affleck, and D. J. Scalapino, Phys. Rev. B 65, 165122 (2002).

[16] M. Yamanaka, M. Oshikawa, and I. Affleck, Phys. Rev. Lett. 79, 1110 (1997); P. Gagliardini, S. Haas, and T. M. Rice, Phys. Rev. B 58, 9603 (1998).

[17] D. Poilblanc, E. Orignac, S. R. White, and S. Capponi, Phys. Rev. B 69, 220406(R) (2004).

[18] S. R. White, Phys. Rev. B 72, 180403(R) (2005).

[19] M. Sigrist, T. M. Rice, and F. C. Zhang, Phys. Rev. B 49, 12058 (1994).

[20] details will be given later, G. Roux et al. (in preparation).

[21] Y. Piskunov et al., Phys. Rev. B 72, 064512 (2005). 